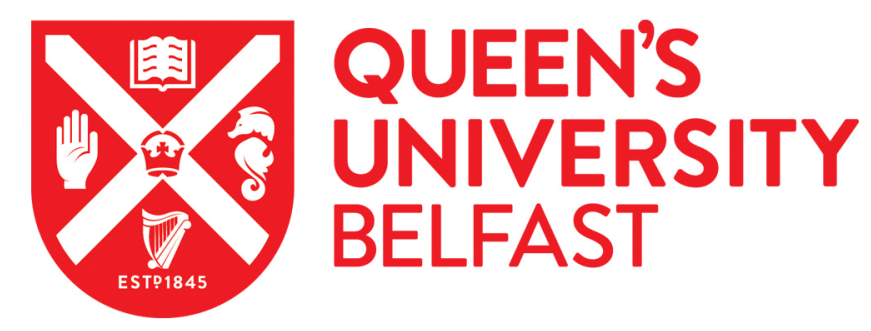

\title{
High harmonics from relativistically oscillating plasma surfaces-a high brightness attosecond source at keV photon energies
}

Zepf, M., Dromey, B., Kar, S., Markey, K., \& Simpson, P. (2007). High harmonics from relativistically oscillating plasma surfaces-a high brightness attosecond source at keV photon energies. Plasma Physics and Controlled Fusion, 49, B149.

Published in:

Plasma Physics and Controlled Fusion

Document Version:

Publisher's PDF, also known as Version of record

Queen's University Belfast - Research Portal:

Link to publication record in Queen's University Belfast Research Portal

\footnotetext{
General rights

Copyright for the publications made accessible via the Queen's University Belfast Research Portal is retained by the author(s) and / or other copyright owners and it is a condition of accessing these publications that users recognise and abide by the legal requirements associated with these rights.
}

Take down policy

The Research Portal is Queen's institutional repository that provides access to Queen's research output. Every effort has been made to ensure that content in the Research Portal does not infringe any person's rights, or applicable UK laws. If you discover content in the Research Portal that you believe breaches copyright or violates any law, please contact openaccess@qub.ac.uk. 
High harmonics from relativistically oscillating plasma surfaces—a high brightness attosecond source at keV photon energies

This article has been downloaded from IOPscience. Please scroll down to see the full text article.

2007 Plasma Phys. Control. Fusion 49 B149

(http://iopscience.iop.org/0741-3335/49/12B/S14)

View the table of contents for this issue, or go to the journal homepage for more

Download details:

IP Address: 143.117.90.74

The article was downloaded on 08/09/2012 at 11:47

Please note that terms and conditions apply. 


\title{
High harmonics from relativistically oscillating plasma surfaces—a high brightness attosecond source at $\mathrm{keV}$ photon energies
}

\author{
M Zepf ${ }^{1}$, B Dromey ${ }^{1}$, S Kar $^{1}$, C Bellei $^{2}$, D C Carroll ${ }^{3}$, R J Clarke $^{4}$, \\ J S Green ${ }^{2}$, S Kneip ${ }^{2}$, K Markey ${ }^{1}$, S R Nagel ${ }^{2}$, P T Simpson ${ }^{1}$, \\ L Willingale ${ }^{2}$, P McKenna ${ }^{3}$, D Neely ${ }^{4}$, Z Najmudin ${ }^{2}$, K Krushelnick ${ }^{2}$ and \\ P A Norreys ${ }^{4}$ \\ ${ }^{1}$ Department of Physics and Astronomy, Queen's University, Belfast BT7 1NN, UK \\ 2 Blackett Laboratory, Imperial College, London, SW7 2BZ, UK \\ 3 SUPA, Department of Physics, University of Strathclyde, Glasgow, G4 0NG, UK \\ ${ }^{4}$ Central Laser Facility, CCLRC Rutherford Appleton Laboratory. Chilton, Didcot, \\ OX11 0QX, UK
}

Received 6 July 2007

Published 15 November 2007

Online at stacks.iop.org/PPCF/49/B149

\begin{abstract}
An intense laser pulse interacting with a near discontinuous plasma vacuum interface causes the plasma surface to perform relativistic oscillations. The reflected laser radiation then contains very high order harmonics of fundamental frequency and-according to current theory-must be bunched in radiation bursts of a few attoseconds duration. Recent experimental results have demonstrated x-ray harmonic radiation extending to $3.3 \AA$ ( $3.8 \mathrm{keV}$, order $n>3200$ ) with the harmonic conversion efficiency scaling as $\eta(n) n^{-2.5}$ over the entire observed spectrum ranging from $17 \mathrm{~nm}$ to $3.3 \AA$. This scaling holds up to a maximum order, $n_{\mathrm{RO}} 8^{1 / 2} \gamma^{3}$, where $\gamma$ is the peak value of the Lorentz factor, above which the harmonic efficiency decreases more rapidly. The coherent nature of the generated harmonics is demonstrated by the highly directional beamed emission, which for photon energy $h v>1 \mathrm{keV}$ is found to be into a cone angle $\sim 4^{\circ}$, significantly less than that of the incident laser cone $\left(20^{\circ}\right)$.
\end{abstract}

(Some figures in this article are in colour only in the electronic version)

\section{Introduction}

Coherent high order harmonic x-ray generation (HOHG) has the potential to open up the world of physical processes on an attosecond (as) timescale [1-3]. The key to this is converting high power optical laser pulses into broad, phase-locked harmonic spectra extending to multi-keV photon energies - which can be achieved, with unprecedented efficiency and brightness, by reflection off relativistically oscillating plasmas [2,3]. As explained below, the process of 
reflecting light from a relativistically oscillating surface not only leads to a substantial frequency upshift_-from the near infrared to multi-keV x-rays-it also intrinsically reemits the incident electromagnetic wave in the form of as pulse. For highly relativistic surface motion (Lorentz factor $\gamma \gg 1$ ) this process achieves very appreciable efficiency and promises to be a route to producing as pulses with source brightness and pulse energy many orders of magnitude above the current state of the art. Additionally, it is predicted that pulse durations as short as a few as $\left(10^{-18} \mathrm{~s}\right)$ and even extending into the zeptosecond $\left(10^{-21}\right)$ regime should be achievable [2].

The unique properties of such a source has led to the investigation of its potential for use in many exciting applications [1,3,4]. The availability of bright as x-ray pulses will allow the probing of the dynamics and properties of atoms and molecules on temporal scales shorter than that of the period of atomic vibrations, i.e. as resolution of bound-free electronic transitions (e.g. from the $4 p$ state of krypton) [5,6]. The short pulse duration and high focused x-ray intensities possible with HOHG will also facilitate the investigation of new regimes of atomic interactions with radiation, such as high field atomic stabilization [7], where the ionization rate decreases with increasing intensity of ionizing radiation for very high intensities. This effect should be easier to observe with short pulse x-rays since the distortion amplitude of bound electron orbits $A \sim E / v^{2}$ is much less for x-rays than optical radiation ( $E$ : electric field strength, $v$ : frequency). In the high field limit for ionization by optical light $\left(\sim 10^{16} \mathrm{~W} \mathrm{~cm}^{-2}\right) A$ is much greater than the atomic radius, while for $\mathrm{x}$-rays it is much less. As a result it should be possible to reach the stabilization regime using $\mathrm{HOHG}$ resulting in a reduction of the ionization rate as the atom is exposed to increasingly high intensity regimes using x-rays, with electrons instead quivering rapidly in their orbits [7].

Recently HOHG pulse production has been cited as a possible route to achieving the huge intensities required for probing the non-linear quantum electrodynamical (QED) properties of the vacuum, providing a significant intensity boost for existing or imminently anticipated laser technology and highlighting the enormous potential of HOHG [4]. These predictions rely on the fact that the focused harmonic radiation can in principle have a substantially higher intensity, $I_{\max }$, than that of the laser, $I$, as discussed below. For example, an incident intensity of $\sim 10^{22} \mathrm{~W} \mathrm{~cm}^{-2}$ could be refocused to $>10^{29} \mathrm{~W} \mathrm{~cm}^{-2}$ corresponding to the critical Schwinger limit [8,9] electric field of $\sim 10^{16} \mathrm{~V} \mathrm{~cm}^{-1}$ for electron-positron pair production from the vacuum [10] - although achieving the required focusing accuracy is technically extremely challenging and the route of using a suitably shaped target as yet experimentally unproven [8].

It should be pointed out that HOHG is not the only route to achieving as pulses. Indeed the field of attoscience has been opened up by using high harmonic generation (HHG) in gases. Harmonic conversion takes place in an extended gaseous medium and has been demonstrated to achieve high conversion efficiency for soft $\mathrm{x}$-rays $(20-100 \mathrm{~nm})$ and as duration pulses [11]. The two harmonic generation techniques appear to be essentially complementary, with HHG suited to small, high repetition rate lasers and the generation of more modest pulse energies at longer wavelengths, while HOHG requires outstanding laser performance in terms of peak power, focusability and contrast and provides higher conversion efficiency (when compared with current HHG results) and scales to shorter wavelengths. The challenge in terms of generating isolated as pulses is essentially very similar in both cases.

The structure of this paper is as follows. First we discuss the underlying physics of HHG from relativistically oscillating surfaces and the origin of the scaling law that govern their behaviour in the limit of very high intensities and its relevance to the production of the as pulses. We then consider the implications for experiments with regard to the required laser performance and finally review the latest results on the source scaling and properties. 


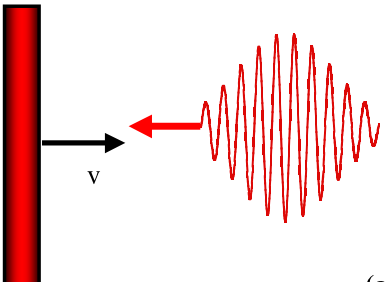

(a)
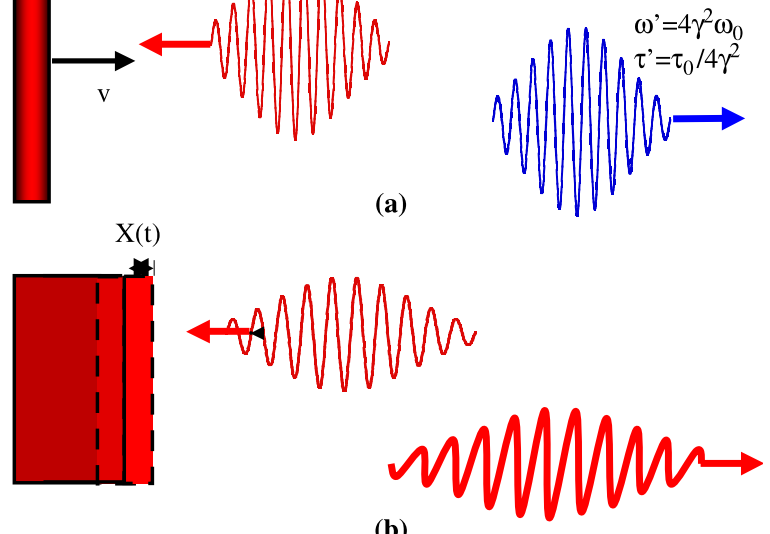

(b)

Figure 1. Schematic of the relativistic upshift of light reflected by a mirror moving with constant $\gamma$ $(a)$. In this case the entire pulse is upshifted and compressed by a constant factor of $4 \gamma^{2}$ and $(b)$ the scenario of relativistically oscillating plasma with an amplitude $X(t)$, which results in periodic compression of the incident EM energy and hence a non-sinusoidal reflected waveform.

\section{Background}

HOHG [12-15] essentially results from an oscillatory extension to Einstein's prediction for the frequency up shift of light reflected off a perfect mirror moving at relativistic velocitiesthe relativistic Doppler effect [16]. In this theory a pulse of duration $\Delta t$ at frequency $\omega_{0}$ is shifted to a frequency of $\omega^{\prime}=4 \gamma^{2} \omega_{0}$ (with the Lorentz factor $\gamma \gg 1$ ). Since the number of cycles in an electromagnetic pulse is a Lorentz invariant the pulse is also compressed by a commensurate amount and the resultant pulse duration is $\Delta t^{\prime}=\Delta t / 4 \gamma^{2}$ (figure 1) [16]. From this one can see that in principle it should be possible to achieve substantial frequency upshifts and extremely short pulses by reflecting a high power laser pulse of a mirror with suitable properties. The key difficulty is, of course, to create a reflective structure moving at a velocity close to the speed of light. Various approaches have been studied over the years, including the upshift from ionization fronts [17] and, most recently, electron density waves in plasmas [18]. While promising in principle, these approaches have not yet been demonstrated to produce intense, coherent radiation pulses.

An alternative approach to achieving a mirror with relativistic velocities is to illuminate an initially solid target with an ultra short, intense laser pulse to create a near discontinuous plasma-vacuum boundary. The electric field of the laser can efficiently couple to the plasma surface [19], causing the electrons to oscillate in phase with the laser [1-3, 8, 13-15, 20, 21], thus constituting a relativistic mirror oscillating at the laser frequency $\omega_{0}$. Since the plasma density is higher than the critical density for such a scenario, the plasma acts as an efficient reflector of the incident laser light. In a seminal paper by Paul Gibbon [15] it was shown that in such circumstances the incident laser light is upshifted very efficiently to higher frequencies. The underlying process in the case of a relativistically oscillating mirror is in many ways similar to the process of the relativistic Doppler upshift described by Einstein [16]. The main difference is that instead of a constant value of $\gamma$ describing the motion of the mirror surface, one now has Lorentz factor that is a function of time $\gamma(\mathrm{t})$.

As the position of this mirror surface is a temporal function of the incident optical laser cycle, the phase of the reflected light wave is modulated such that it is no longer sinusoidal 


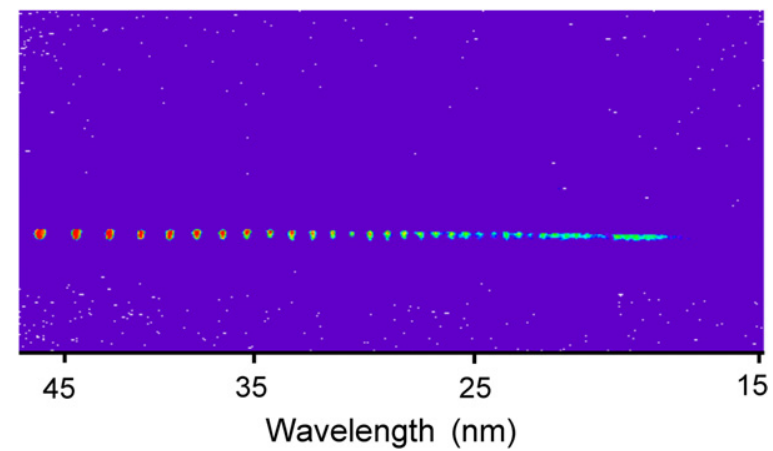

Figure 2. Typical spectrum of high harmonics obtained with a $1 \mathrm{ps}$ duration pulse at $1 \mu \mathrm{m}$ wavelength at $I \sim 10^{19} \mathrm{~W} \mathrm{~cm}^{-2}$ (from [23]).

as shown in figure $1(b)$. As can be understood from Fourier theory, such a waveform must contain many high order harmonics of the fundamental frequency. The initial theoretical approach — a physical picture first proposed by Bulanov et al [13]—was therefore to describe the harmonics observed in PIC simulations in terms of the reflection of the incident laser off a moving mirror oscillating at the laser frequency $\omega_{0}$. A detailed semi-analytical moving mirror model was developed by Lichters et al and was found to be in good agreement with PIC simulations [14,22]. This demonstrated that the picture of the moving mirror captures the essence of the harmonic generation process. Experiments performed in the mid-1990s observed harmonic spectra (figure 2), where the conversion efficiency $\eta(n)$ of a given harmonic order $n$ followed a power-law scaling $\eta(n) \sim n^{-P}$, where $P$ is an intensity dependent exponent that increased from $P=5.5$ to $P=3.3$ when the intensity was varied from $5 \times 10^{17}$ to $10^{19} \mathrm{~W} \mathrm{~cm}^{-2}$ [23].

However, since the surface trajectory was not calculated self-consistently, the moving mirror model provides qualitative rather than quantitative information about the harmonic generation process. In particular, this model did not have any predictive power as to either the ultimate efficiency, or limiting value of $P$ that might be expected in the limit of very large laser intensities or the ultimate extent of the harmonic spectrum towards high frequencies.

The most recent theoretical development in the field by Gordienko et al and Baeva et al $[2,20]$, elegantly based on similarity theory, identifies the sharp spikes in the temporal variation of the Lorenz factor $\gamma$ as the key to the production of the highest harmonics. That this should be the case can be easily understood when one considers the temporal variation of $\gamma(t)$. Even assuming a very smooth variation of the actual surface velocity with time (e.g. $v(t) \sim \sin (\omega t)$ as in figure 3$)$ the corresponding variation of $\gamma(t)$ is sharply spiked. From Einstein's theory of relativistic Doppler upshift one would therefore expect the upshifting process to be restricted to a timescale of the order of the temporal width of each ' $\gamma$-spike'substantially shorter than an optical half cycle - and the maximum upshift to take place when the Lorentz factor reaches its maximum $\gamma_{\max }$.

The key finding is that the harmonic spectrum assumes an asymptotic spectral shape in the so-called relativistic limit (where $\gamma_{\max } \gg 1$ ). The spectrum retains a power-law scaling for the conversion efficiency in the relativistic limit with the efficiency of the $n$th harmonic reaching

$$
\eta(n) \sim n^{- \text {Prel }}
$$



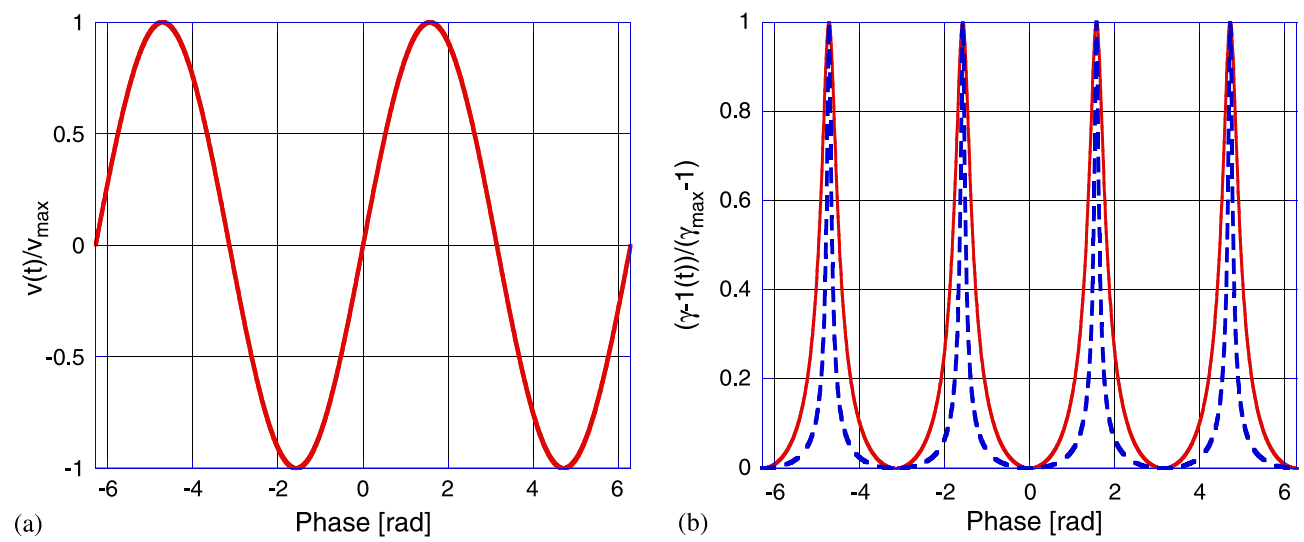

Figure 3. The Lorentz factor $\gamma$ displays sharp spikes even for a very smooth variation of velocity with time. Here a simple sinusoidal velocity dependence $\left[v(\phi)=v_{\max } \sin (\phi)\right]$ is chosen to illustrate the relative behaviour of $v(t)$ and $\gamma(t)$. Plot $(b)$ shows the variation of the Lorentz factor for two different peak velocities $v_{\max }=0.995 \mathrm{c}\left(\gamma_{\max }=5.7,-\right)$ and $v_{\max }=0.985 \mathrm{c}\left(\gamma_{\max }=22.3\right.$, - - - ). Note that the width of the $\gamma$-spikes are substantially narrower than the oscillation period and the FWHM of the $\gamma$-spikes reduces linearly with increasing $\gamma_{\max }$.

with $P_{\text {rel }}=8 / 3$ [20]. Another important result of this theory is the prediction of the highest harmonic where the equation (1) still applies up to an order

$$
n_{\mathrm{RO}} \sim 8^{1 / 2} \gamma_{\max }^{3}
$$

beyond which the conversion efficiency decreases more rapidly or 'rolls over' (where $\gamma_{\max }=$ $\left(1+3.6 \times 10^{-19} I \lambda^{2}\right)^{1 / 2}$ corresponds to the maximum surface velocity, $I\left(\mathrm{~W} \mathrm{~cm}^{-2}\right)$ is the peak intensity and $\lambda(\mu \mathrm{m})$ the wavelength of the laser). This is in contrast to the moving mirror model, where one would anticipate a cut-off or at least a rapid decrease in efficiency for harmonic orders $n>4 \gamma_{\max }^{2}$ [8]. The physical origin of this substantially larger frequency upshift also derives directly from the $\gamma$-spikes. Since the emission of high harmonic orders only takes place for large values of $\gamma$ a sharp temporal localization of the emitted harmonics results. As illustrated in figure 3, the temporal duration of the $\gamma$-spikes reduces for increasing intensity as $T_{\text {Spike }} \sim T_{0} / \gamma_{\max }$ (with $T_{0}=2 \pi / \omega_{0}$ ) [20]. The pulses of duration $T_{\text {spike }}$ are upshifted and compressed by a factor of $4 \gamma_{\max }^{2}$ - familiar from the relativistic mirror. As a result the harmonics are emitted in short temporal bursts with $T_{\text {burst }} \sim T_{\text {Spike }} / \gamma_{\max }^{2} \sim T_{0} / \gamma_{\max }^{3}$ and hence, from Fourier Theory, must contain significant spectral components up to frequencies of $\mathrm{O} \sim \omega_{0} \gamma_{\max }^{3}$. In effect, the surprising new result of the theory of relativistic spikes is that the high energy cut-off and the ultimate slope of the spectrum is governed by the temporal compression and truncation of the electromagnetic pulse rather than the maximum upshift expected from a relativistic mirror moving at constant $\gamma$.

\section{Attosecond pulse production and harmonic beam intensity}

The fact that the harmonics extend to substantially higher frequencies than would be expected from the conventional moving mirror model is therefore a direct result of the as temporal bunching of the emitted harmonics-and consequently an observation of the $n_{\mathrm{RO}} \sim 8^{1 / 2} \gamma_{\max }^{3}$ scaling would be evidence for the as temporal bunching of the harmonic emission. It is this temporal compression of the incident electromagnetic energy by the HOHG process that 

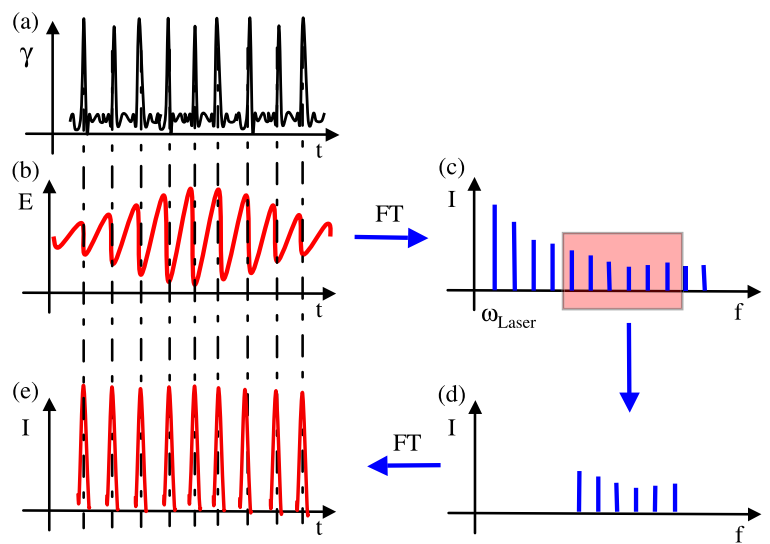

Figure 4. Schematic of the harmonic generation process. The periodic spiking of the $\gamma$-factor (a) leads to phase modulation of the reflected laser radiation $(b)$ and the corresponding spectrum (c) exhibits harmonics following a power-law decay to very high orders. Filtering out a number of harmonics as indicated in $(c)$ and $(d)$ results in the formation of an as pulse train $(e)$, or under suitable conditions, an isolated as pulse.

provides the first part of the condition necessary for the as pulse generation. As this occurs twice every laser cycle the emission is expected to be a train of pulses [20].

However, the reflected waveform itself, as indicated in figures $1(b)$ and 4 is a superposition of the reflected laser light and its higher harmonics. Figure 4 illustrates the effect of the reflection of the oscillating surface on the reflected waveform. Since most lasers have pulse durations corresponding to more than one optical cycle the Fourier transform of the reflected waveform exhibits distinct harmonics of the laser frequency. Since the laser frequency $\omega_{0}$ is the strongest spectral component, the isolation of the as pulses requires the use of filters to reject the laser frequency [3]. In the typical case of a multi-cycle laser the introduction of a filter, as indicated in figure $4(c)$, results in a train of as pulses being produced. In relativistic limit and using an ideal high-pass filters, the shortest possible duration of the as pulses is determined by the dominant frequency component transmitted by the filter, and consequently filters that only transmit the highest harmonic orders will lead to the production of the shortest possible pulses (more generally, the exact filter transmission curve must be taken into account).

To exploit the full potential of as duration pulses, one would prefer to produce single, isolated as pulses rather than the pulse train indicated in figure 4(e). Such pulses can be produced in principle by using laser with a pulse duration that corresponds to only one optical cycle [3]. The rapid variation of the pulse envelope results in the highest harmonics only being produced during one laser half cycle and consequently the production of only one isolated as pulse of duration $\sim T_{0} / \gamma^{3}$ is achieved with appropriate filtering. In this case the Fourier transform in figure 4(c) no longer exhibits individual harmonic peaks, but instead becomes a continuum with a power-law decay $\eta(\omega) \sim \omega^{-P}$.

Note that the conversion efficiency into a single as pulse is much higher than that for an individual harmonic, since the spectral width spans many harmonic orders [3]. The maximum number of harmonics $\Delta n$ contributing to an as pulse with a fixed fractional bandwidth at a given central frequency $n_{\mathrm{cf}} \omega_{0}$ scales linearly with the central frequency so that $\Delta n=k n_{\mathrm{cf}}$, where $\mathrm{k}$ is a factor $\mathrm{O} \sim 1$ which depends on the filter transmission function and the exponent governing the harmonic efficiency $P$. The conversion efficiency into a given as pulse is therefore $\eta_{\text {att }} \sim n_{\mathrm{cf}}^{-8 / 3} \times \Delta n \sim n_{\mathrm{cf}}^{-4 / 3}$ and decays much more slowly than the conversion efficiency into a given harmonic. Detailed calculations for realistic filters and laser intensities 
show that the conversion efficiency into an 84 as duration pulse centred at $20 \mathrm{eV}$ photon energy could reach several per cent of the incident laser energy and still approach $0.1 \%$ for a 5 as pulse centred at $400 \mathrm{eV}$ [3]. Such a performance would be truly spectacular compared with current short pulse $\mathrm{x}$-ray sources.

It should be mentioned that the production of isolated as pulses can also be achieved using laser pulses with pulse durations corresponding to multiple laser cycles. Such schemes rely on suppressing the harmonic production in all but one optical half cycle, for example by utilizing pulses with a complex polarization state [20,24].

Another exciting conclusion that can be derived from theoretical considerations and simulations is that the reflected harmonic radiation can, in principle, be refocused to higher intensities than that of the incident laser [2]. While this result may be initially surprising it is the direct result of the temporal compression of the reflected electromagnetic energy and the - in principle - enhanced focusability of the short wavelength radiation. Based on a value of $P_{\text {rel }}=8 / 3$, one obtains a conversion efficiency for pulse generation that depends on the central frequency as $\sim n_{\mathrm{cf}}^{-5 / 3}$. When this is coupled with the increased focusability $\left(\sim n_{\mathrm{cf}}^{2}\right)$ and temporal compression of the reflected energy $\left(\sim n_{\mathrm{cf}}\right)$ one obtains a peak intensity that scales as $I_{\max } \sim I_{0} \cdot n_{\mathrm{cf}}^{4 / 3}$. These scalings serve to highlight the exceptional potential of this XUV and $\mathrm{x}$-ray source. While one might express justified reservations as to the smallest refocused spot-size that could be achieved in practice, this scaling still serves to highlight that $\mathrm{x}$-ray intensities far beyond the previous limits are within reach.

\section{Competing mechanisms of harmonic generation}

The interaction of an intense laser pulse with a steep, overdense plasma is quite complex and so far we have chosen to discuss only one facet of the interaction-the production of high harmonics by reflection of a relativistic mirror. Many other phenomena of interest, absorption and energy transport, particle acceleration take place in parallel and are discussed at some depth in the literature (e.g. [25] and references therein). From the point of view of harmonic production a competing process of harmonic production by so-called coherent wake emission (CWE) [26] requires a brief mention. Briefly, these harmonics result from the periodic pumping of the plasma waves at the steep plasma-vacuum interface and decay by radiating harmonics of the laser frequency and were first investigated using high power $\mathrm{CO}_{2}$ lasers in the late $1970 \mathrm{~s}$ and early 1980s [12]. Consequently the highest harmonic that can be generated by this process must have a frequency that is no higher than the maximum plasma frequency $\omega_{\mathrm{p} \_ \text {max }}$ in the density ramp, i.e. $n_{\max }=\omega_{\mathrm{p} \_ \text {max }} / \omega_{0}$. This process scales linearly with the energy transferred to the hot electrons in the interaction, and thus almost linearly with laser intensity. For values of $I \lambda^{2}$ as high as the mid- $10^{18} \mathrm{~W} \mathrm{~cm}^{-2} \mu \mathrm{m}^{2} \mathrm{CWE}$ harmonics dominate the observed harmonic spectra and are observable at intensities as low as $10^{16} \mathrm{~W} \mathrm{~cm}^{-2}$.

\section{Experimental considerations}

From the previous discussion of the theory it is clear that in order to achieve the predicted conversion efficiency and performance a relativistic mirror of extremely high quality must be created. In practical terms one requires a well-defined reflection point for the incoming laser, i.e. the reflection of the laser should take place over a length that is small compared with the incident wavelength to prevent destructive interference from waves reflected at different positions from dramatically reducing the harmonic efficiency. In the case of step plasma, this is trivially the case and the reflection process is localized over a length on the order of the skindepth 
$c / \omega_{\mathrm{p}}$. In the case of plasmas with a much longer (shallower) density scalelength $L$ the light propagates into an extended refractive index gradient and the reflection of electromagnetic energy is no longer well localized and substantially reduced efficiency, particularly for the higher orders, is observed. From these considerations it is clear that ideally the scalelength $L$ should be short enough not to appreciably increase the length scale over which the reflection takes place. The issue trade-off between harmonic generation efficiency and plasma scalelength has been studied using PIC simulations and experimentally $[14,19]$ and it was found that $L>0.2 \lambda$ results in substantially reduced efficiency. However, it also appears that perfect step profiles result in reduced efficiency [14]. This latter effect was attributed to greater plasma restoring forces, making the plasma stiffer and thus leading to smaller amplitude oscillations for a given laser intensity. In practice, however, achieving $L<0.2 \lambda$ with a peak intensity of $10^{20} \mathrm{~W} \mathrm{~cm}^{-2}$ or above presents a greater challenge. Given typical plasma expansion velocities, this implies that any plasma formation must be suppressed for all times greater than a few ps from the peak of the pulse - a formidable challenge for laser engineering. Typical high power lasers exhibit an intensity contrast of around $10^{7}: 1$ between the amplified spontaneous emission and the peak of the pulse on timescales of $100 \mathrm{~s}$ of ps before the pulse peak. This implies that for intensities above the mid $10^{18} \mathrm{~W} \mathrm{~cm}^{-2}$ any further increase in intensity leads to the plasma forming at increasingly early times and hence a longer scalelength plasma being formed. In practical terms one finds that once the prepulse pedestal is above the plasma formation threshold, the harmonic efficiency begins to decrease with increasing peak laser intensity, due to the detrimental effect of a long scalelength.

The above scenario also explains why historically experiments using longer pulses ( $500 \mathrm{fs}-$ $1 \mathrm{ps)}[23,27]$ have observed very high order harmonics in good agreement with simulations, while replicating these results with similarly intense, short pulse lasers ( $<150 \mathrm{fs}$ ) has yet to be accomplished. The difference is simply that significant plasma density profile modification due to the ponderomotive pressure is possible on a ps timescale [28]. Hence a ps laser which has a contrast resulting in a reasonably shallow density profile of, say, $L=\lambda$ just before the arrival of the pulse peak can still be steepened sufficiently by the ponderomotive pressure to allow efficient harmonic generation. Conversely, for much shorter pulses, the profile modification becomes negligible and the peak of the pulse essentially interacts with the density scalelength created by the prepulse.

Clearly another area in which utmost care must be taken is the spatial quality or flatness of relativistic mirror. Any substantial deviations from an ideal mirror will inevitably lead to distortions of the reflected wavefront. Under ideal conditions, the reflected harmonics are expected to be diffraction limited [29]. That this should be the case can be understood by considering the scenario of relativistic upshift from a mirror moving at constant velocity. As long as the mirror is flat enough to reflect the incident radiation without distortion for $v=0$, this will also be the case for $v \sim c$. Hence for $v \sim c$ the reflected radiation is substantially upshifted in frequency and hence will have a much smaller diffraction limited divergence. However, one would anticipate that a rough, uneven target surface would result in diffuse reflection and therefore much broader angular distribution.

While using a target with an initial target roughness on the Angstrom scale is easily possible by using highly polished targets, controlling the plasma surface requires special consideration. Once a plasma has formed, any uneven expansion will lead to a distorted surface and hence be detrimental to the angular distribution of the reflected harmonics. More critically, the modification of the plasma profile due to the ponderomotive pressure of an intense laser pulse has been shown to lead to denting of the plasma surface in the early phase, followed by severe, Rayleigh Taylor-like surface modulations [28]. This implies that, while it is easier to reach sufficiently steep density profiles using longer pulses, the ponderomotive effects also result in 


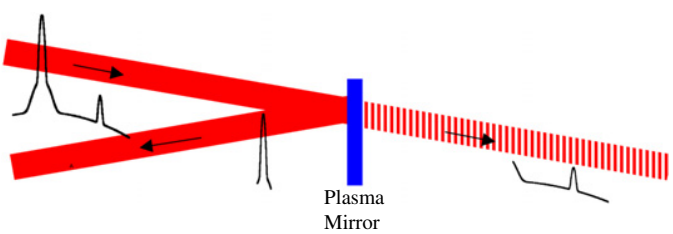

Figure 5. Schematic of a plasma mirror set-up used to enhance the pulse contrast of high power lasers. The laser is incident on a low reflectivity substrate that transmits the prepulse, while the main pulse is reflected by the high reflectivity plasma that forms on the mirror surface just prior to the arrival of the pulse peak.

surface instabilities which are detrimental to the angular distribution of the harmonics. This interpretation is in excellent agreement with the earlier work [23], where very high harmonic orders were observed with an essentially isotropic angular distribution. In these experiments with ps pulses, the intense ponderomotive pressure leads to both significant steepening of the initial density profile and the development of a substantially distorted critical density surface.

The growth of these instabilities can be estimated simply from momentum conservation considerations, i.e. the momentum required to achieve a certain amount of surface motion must be balanced by the laser momentum $p_{\mathrm{L}}=2 I / c$. From this it is clear that using shorter pulses and higher density targets will minimize any surface distortion. In the limit of a few cycle pulses, dynamic surface distortions can be effectively ignored for all practical purposes.

From the above discussion it is clear that achieving high contrast is a key requirement for this type of experiment. In order to be able to investigate harmonics with laser currently available the pulse contrast needs to be improved substantially. This is typically done using a technique called the plasma mirror [30,31], whereby a low reflectivity surface (e.g. antireflection coated glass with $R_{0}<1 \%$ ) is placed in the laser beam so that the peak of the pulse exceeds the plasma formation threshold, but the entire prepulse at times greater than $\sim 1$ ps is below the plasma formation threshold. This results in the peak of the pulse being reflected by the plasma and the prepulse being transmitted by the low reflectivity substrate (figure 5). The efficiency of the process is governed by the plasma reflectivity (typically $R_{\text {plasma }}=0.5 \ldots 0.8$ ) and the contrast is improved by a factor of $F=R_{\text {plasma }} / R_{0} \sim 100$ per plasma mirror. The typical rise-time of the plasma mirror is $\sim 150 \mathrm{fs}$ or less, making the plasma mirror an ultra-fast switch capable of substantially improving intrinsic laser contrast. The main disadvantage is the low efficiency - particularly when multiple surfaces are employed-and the fact the plasma mirror surface is destroyed on each shot.

\section{Recent experimental advances: scaling of efficiency, maximum frequency up shift and angular distribution}

While the potential of harmonics from solid targets has been recognized for some years [15,23], the experimental investigation has been limited by the available lasers. In particular, testing the ultimate conversion efficiency and highest harmonic orders that can be generated are very important steps in terms of understanding the underlying physics and developing the source.

The Vulcan Petawatt laser at the Rutherford Appleton Laboratories [32] is ideally suited for generating harmonic radiation as it can readily reach the desired peak intensities of $\sim 10^{21} \mathrm{~W} \mathrm{~cm}^{-2}$, delivering up to $\sim 600 \mathrm{~J}$ on target in $\sim 500 \mathrm{fs}$. However, with an intrinsic prepulse pedestal that extends for $\sim 5 \mathrm{~ns}$ at a contrast of $10^{7}: 1$, it is unsuitable for the formation of the short plasma scalelength, $L<0.2 \lambda$, required for efficient harmonic generation [19], where $\lambda$ is the laser wavelength. As a result the contrast of the incident pulse was increased 

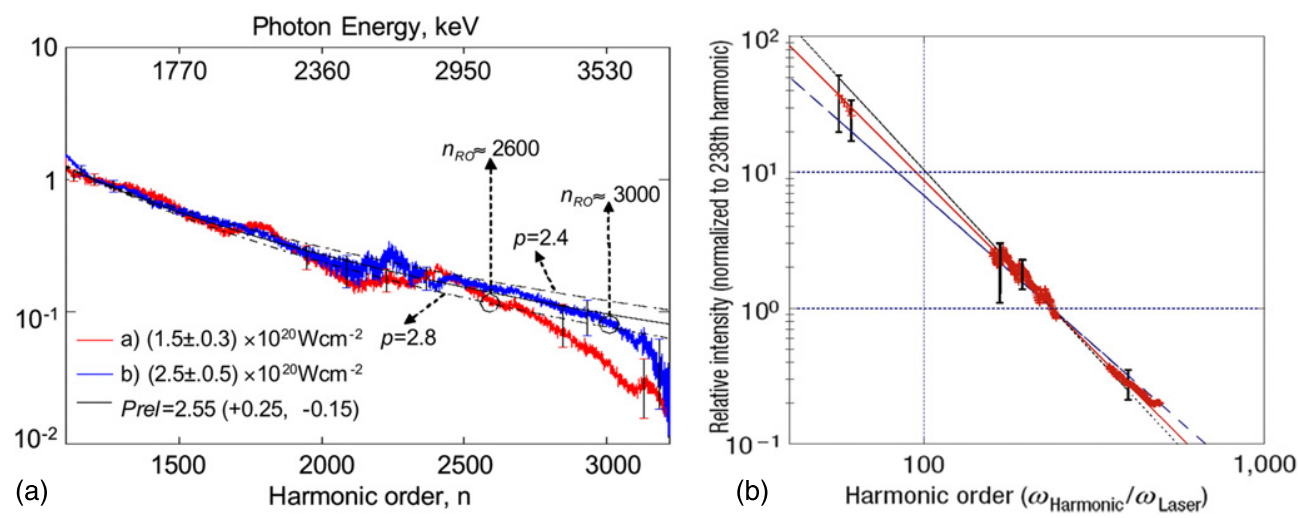

Figure 6. Power-law scaling in the relativistic limit obtained on the Vulcan Petawatt in the x-ray regime (plot $(a)$, 1200th-3200th order, 9-3 A) and the XUV (plot (b), 60th-850th orders, 17-3 nm). The x-ray harmonic spectra were taken for two different intensities: $(1.5 \pm 0.3) \times 10^{20} \mathrm{~W} \mathrm{~cm}^{-2}$ (red trace) and $(2.5 \pm 0.5) \times 10^{20} \mathrm{~W} \mathrm{~cm}^{-2}$ (blue trace). The lines are fits to the data such that $I(n) / I(m)=n^{-P} / m^{-P}$, where $p$ is the fitting parameter and $m$ is the harmonic order to which the spectrum is normalized $(m=1200$ in $(a)$ and $m=238$ in $(b))$. The best fit $(-)$ is for a value of $P_{\text {rel }}=2.55(+0.25,-0.15)$ in $(a)$ and $P_{\text {rel }}=2.5(+0.2,-0.3)$ in $(b)$ and is consistent with that expected for harmonic generation in the relativistic limit. The dashed lines in $(a)$ represent $p=2.4$ and $p=2.8$, while in $(b)$ the upper and lower bounds correspond to $p=2.2$ and 2.7 respectively. Error bars represent the uncertainty in the relative signal strength arising from the detector and filter transmission taking into account the individual uncertainty in each of the relevant quantities. The absence of discrete harmonics in the spectra is due to the limited resolution of the crystal spectrometer used.

to $>10^{10}: 1$ at $\sim 10 \mathrm{ps}$ from the peak of the pulse by the use of a double plasma mirror setup $[27,30,31]$ to achieve the required sharp plasma-vacuum interface.

The laser focus size was routinely monitored by imaging the size of the emission region at $3 \omega_{\text {Laser }}$ to ensure high intensity both with and without plasma mirrors [33]. The x-ray signal (keV range) was observed using a broadband crystal spectrometer consisting of mica crystal in a von Hamos geometry [33] and a Fujifilm 'image plate' detector (BAS with $9 \mu \mathrm{m}$ $\mathrm{CH}$ protective layer) [34]. The point spread function of the spectrometer was experimentally determined and the resolution $(\lambda / \Delta \lambda)$ of the spectrometer was determined to be $\sim 200$ (limited by the crystal bending inaccuracies). The XUV harmonics in the range $17-3 \mathrm{~nm}$ was detected using a Hitachi flat-field grating with 1200 lines $\mathrm{mm}^{-1}$ and detected using an ANDOR CCD detector. This spectrometer employed a curved mirror to collect the light transverse to the direction of dispersion.

Angular distribution measurements in the $\mathrm{keV}$ range were performed using pieces of 'image plate' giving data over a $2 \pi$ range. Differential filtering $(5 \mu \mathrm{m} \mathrm{Mg}, 5 \mu \mathrm{m}$ and $15 \mu \mathrm{m} \mathrm{Al})$ was used for signal discrimination. The observed signal levels were consistent only with $\mathrm{x}$-ray emission. The possibility of low energy electrons significantly contributing to the signal was eliminated by the inclusion of deflecting magnets. The data point exactly on the specular direction is taken from the $\mathrm{x}$-ray spectrometer.

The predicted slow decay of the conversion efficiency to higher orders has important consequences for harmonics from relativistic surfaces, since the exact slope determines the conversion efficiency and pulse duration of an as pulse generated by filtering the reflected light [3]. The slow slope is also key to the potential increase of the focused intensity with increasing harmonic order.

Figure 6 shows the recently obtained experimental observations [27] confirming HOHG efficiency scaling in the relativistic limit in the $17-4 \mathrm{~nm}$ spectral range. The experimentally 
obtained value of $P_{\text {rel }}=2.5(+0.2 /-0.3)$ is in agreement with the prediction of $P_{\text {rel }}=8 / 3$. The question remains as to whether this efficiency scaling does indeed extend to the very high harmonic orders required to produce intense as pulses at Ångstrom wavelengths.

Harmonic spectra from smooth $\mathrm{CH}$ targets (few nm surface roughness) and under high contrast conditions, in the $\sim 9 \AA-3.3 \AA$ spectral region, are presented in figure $6(a)$. These demonstrate for the first time that harmonics with $\mathrm{keV}$ photon energies can indeed be generated using this technique. The observed spectrum is consistent with the predicted efficiency scaling equation (1) in the relativistic limit $[20,27]$ up to $\sim 2600$ th $(\sim 3 \mathrm{keV})$ order for an incident intensity of $(1.5 \pm 0.3) \times 10^{20} \mathrm{~W} \mathrm{~cm}^{-2}$, and up to $\sim 3000$ th order $(\sim 3.5 \mathrm{keV})$ for $(2.5 \pm 0.5) \times 10^{20} \mathrm{~W} \mathrm{~cm}^{-2}$. At the highest intensity the observed spectra extend to $\sim 3.8 \mathrm{keV}$, or the 3200 th order of $1055 \mathrm{~nm}$ fundamental $(3.3 \AA$ ) before dropping to the noise floor of the detector. This is the first ever observation of $>1000$ th order from any harmonic generation process and is substantially higher than the previous best results from solid targets (75th order [23], 850th order [27]).

The relative signal strengths shown in figure 6 were obtained by correcting the detected signal for the transmission function of the spectrometer (mica crystal or grating reflectivity, filters and detector response).

The relativistic exponent derived from the $\mathrm{keV}$ data is $P_{\text {rel }}=2.55(+0.25,-0.15)$, demonstrating the slow decay to high orders that is essential for efficient as production and in excellent agreement with the slope observed at longer wavelengths and the predicted slope of $P_{\text {rel }}=8 / 3$. The experimentally observed signal is consistent with harmonic energies of $\sim 17 \mu \mathrm{J}$ at 1200 th $(1.4 \mathrm{keV})$ and $\sim 5 \mu \mathrm{J}$ at 2600 th $(3.1 \mathrm{keV})$ in $1 \%$ bandwidth, respectively. This is comparable to the state of the art at even the largest facilities for coherent $\mathrm{x}$-ray generation at such energies.

At the highest orders the efficiency scaling is characterized by a departure from the scaling given by equation (1). The exact position of this roll-over is found to be intensity dependent and is defined here as the point where the fitting parameter $p>2.8$ relative to $n=1200$. Using this definition we obtain $n_{\mathrm{RO}} \sim 2600$ and $\sim 3000$ for incident intensities of 1.5 and $2.5 \times 10^{20} \mathrm{~W} \mathrm{~cm}^{-2}$, respectively. For our experimental conditions we estimate $\gamma=10 \ldots 13$ based on the intensity of the laser. This would correspond to a value of $n_{\mathrm{RO}} \sim 500$ based on the roll-over at $n_{\mathrm{RO}} \sim 4 \gamma^{2}$ derived from the model for relativistic Doppler shift, suggesting that a departure from the $n^{- \text {Prel }}$ scaling should be visible on the XUV spectrum in figure 6(a). Instead from the X-ray data presented in figure $6(b)$, it is clear that the onset of this rollover regime begins at much higher orders with $n_{\mathrm{RO}}>3000$ for $(2.5 \pm 0.5) \times 10^{20} \mathrm{~W} \mathrm{~cm}^{-2}$. These measurements are in good agreement with most recent theory [20], which predicts $n_{\mathrm{RO}} \approx 8^{1 / 2} \gamma^{3}$, based on interpreting the $\mathrm{x}$-ray harmonic generation process as being due to the sharp spikes in the relativistic $\gamma$-factor of the plasma surface described above. As shown in figure 7 the dependence of $n_{\mathrm{RO}}$ is also consistent with the highest observed harmonics from previous measurements [23].

Based on the results presented here and current theory, it should in principle be possible to generate $10 \mathrm{keV}$ zeptosecond pulses [2] with a conversion efficiency of $>10^{-7}$ using intensities $\sim 7 \times 10^{20} \mathrm{~W} \mathrm{~cm}^{-2}$ - a level readily achievable with the current generation of lasers.

For high pulse contrast and smooth (few nm surface roughness) $\mathrm{CH}$ targets, the HOHG signal $>1 \mathrm{keV}$ is observed to be emitted into a cone angle $\sim 4^{\circ}$ full width half maximum (FWHM) with a low-intensity halo of $\sim 13^{\circ}$ (figure 8 ). This narrow beaming of the harmonic emission is strong evidence of the coherent nature of the harmonic production process. Depending on the exact harmonic source size one would expect a diffraction limited beam at $1 \mathrm{keV}$ to have a divergence that is about $100 \times$ less than that observed experimentally. 


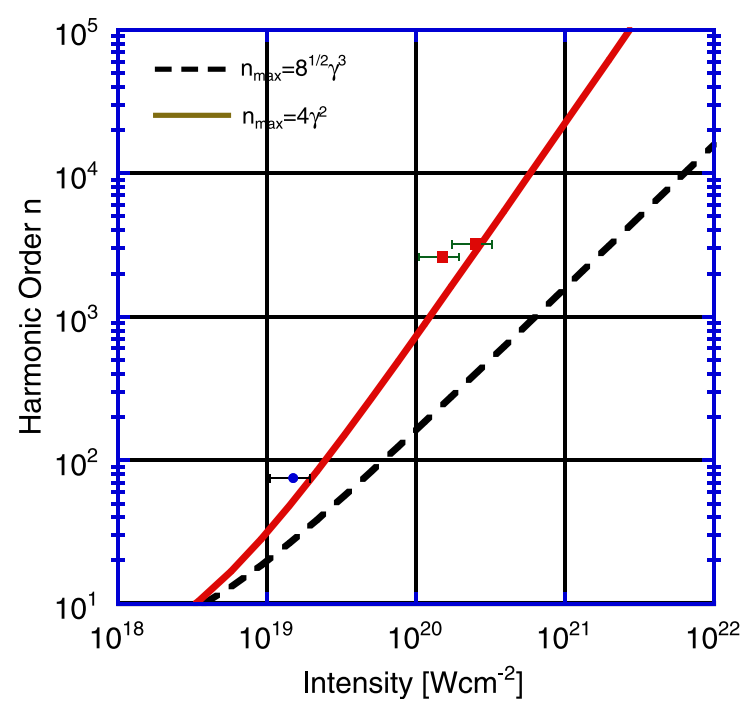

Figure 7. Comparison of the $8^{1 / 2} \gamma^{3}$ (—) and $4 \gamma^{2}(-\ldots)$ scaling for the maximum relativistic limit harmonic order, $n_{\mathrm{RO}}$, with experimentally observed data. The $8^{1 / 2} \gamma^{3}$ scaling is clearly a better fit to the highest observed harmonic from previous experiments (circle from [23], and the two values of $n_{\text {RO }}$ presented in this paper, squares). The rapid scaling of $n_{\text {RO }}$ with intensity allows the extension of atto-and zeptosecond pulse generation to sub-Angstrom wavelengths with realistic lasers.

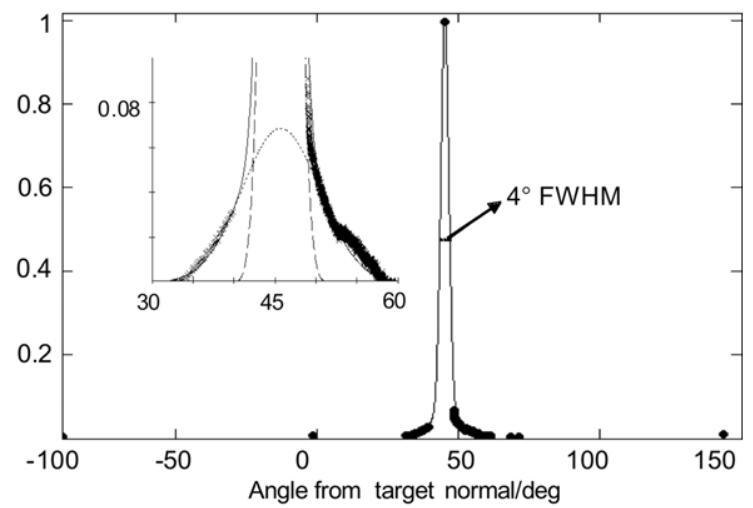

Figure 8. Angular distribution of $>1 \mathrm{keV} x$-ray signal under high contrast conditions. The signal is emitted into a narrow cone peaked in the specular direction at $45^{\circ}$ (the laser incidence angle is minus $\left.-45^{\circ}\right)$. The inset shows a $13^{\circ}$ FWHM Gaussian fit to the scattered $x$-ray halo $(\cdots \cdots)$ and the $4^{\circ}$ fit to the strongly peaked HOHG signal (- - -). The FWHM of the summed double Gaussian fit to the signal is $\sim 4^{\circ}$ (—), which is considerably less than the $f / 3$ laser cone angle $\left(20^{\circ}\right)$. It also demonstrates the absence of significant laser induced surface modulation present in previous, lower contrast experiments [23].

Given the high intensity and pulse duration in this experiment some surface deformation is unavoidable [28]. We estimate that we are currently in the 'denting' regime described above (due to the steep density gradient and high maximum density present in our interactions the instability growth is much slower than in [28]). Therefore the harmonic beam is not reflected from a flat surface, but from a concave surface and hence the divergence of the harmonic beam is expected to be significantly affected by the surface curvature. 
This $4^{\circ}$ cone is consistent with harmonics being reflected from a surface with a small amount of curvature induced by the laser ponderomotive pressure. Assuming that the beam divergence is primarily determined by surface curvature, it is possible that the observed beam corresponds to a near diffraction limited, highly focusable beam.

However, it is also possible that the angular distribution is the result of an imperfect phase distribution of the laser beam. This is analogous to the situation observed when frequency doubling lasers in nonlinear crystals, the absolute wavefront error can be transferred to the harmonic beam, leading to a beam which is then a much higher multiple of the diffraction limit than the original beam. These hypotheses can be tested in future experiments using shorter pulses, which would substantially reduce the surface denting for a given intensity and allow residual effects due to the laser phase to be isolated. However, it should be noted that laser phase errors should not place a fundamental limit on the achievable x-ray beam quality and, as pointed out above, diffraction limited x-ray beams are at least possible in principle using this technique.

The low intensity halo shown in figure 8 is likely due to small surface roughness. Consistent with the result for $\mathrm{keV}$ harmonics, the $3 \omega_{\text {Laser }}$ radiation was only observed with the collection mirror positioned in the specular direction for nm-roughness targets. By contrast, for gold foils with micrometre scale surface roughness, $3 \omega_{\text {Laser }}$ emission was observed both in specular and at $90^{\circ}$ to specular, indicating that the angular distribution is indeed strongly correlated with the shape/roughness of the target surface. No x-ray harmonic signal was recorded for such rough targets. It is assumed that the wide angular distribution due to scattering reduced the signal level to below the noise floor of the $\mathrm{x}$-ray detector.

\subsection{Attosecond pulses}

From both simulations and from the theory of $\gamma$-spikes it is clear that the harmonics must be emitted in the form of an as pulse train or- under special conditions - in the form of a single as pulse. While the direct measurement of the temporal structure of the reflected harmonics has not yet been performed experimentally, the current data already provide indirect confirmation of the as structure of the emitted harmonic pulse-trains. Figure 7 shows that the highest harmonic order observed extends scales with $n_{\mathrm{RO}} \sim 8^{1 / 2} \gamma^{3}$ as opposed to the $4 \gamma^{2}$ scaling one would expect from an ordinary relativistic mirror. The fact that the reflected harmonic radiation contains frequencies which are much higher than that expected due to the relativistic Doppler effect is a direct consequence of the temporal structure of the emitted harmonic radiation. Based on current theory [20] the observed scaling of the highest harmonics observed for a given laser intensity is clear, if indirect, confirmation of the as nature of the emitted radiation pulses.

\section{Conclusion}

Harmonic generation from relativistic surfaces is shown to be a source of extremely high quality $\mathrm{x}$-rays and allows high power lasers to be up shifted to very high photon energies with remarkable efficiency. The observation of a $\gamma^{3}$ dependence of the highest observable harmonic provides clear indication-based on current theory — of the as nature of the emitted harmonic pulses.

Based on the scalings for efficiency and $n_{\mathrm{RO}}$ typical photon numbers (from equation (1)) expected per as/zeptosecond pulse [3] for the given central energies are: $\sim 7 \times 10^{12}$ photons/pulse at $1.5 \mathrm{keV}, \sim 2 \times 10^{12}$ photons/pulse at $2.5 \mathrm{keV}$ and $\sim 8 \times 10^{11}$ photons/pulse at $3.5 \mathrm{keV}$. In this context, these photon numbers appear suitable for the proposed high resolution 
(10 s of Ångstroms) imaging of bio-molecules using ultra-short x-ray pulses [4]. This technique has recently been verified using intense radiation from the FLASH soft-X-ray free-electron laser at $32 \mathrm{~nm}$ for the diffraction limited imaging of a complex nano-structured object [ch]. Based on this, and the results presented here, the prospects for future femto-, atto- and zeptosecond keV x-ray pulses look very bright indeed.

\section{Acknowledgments}

The authors acknowledge the excellent support from the staff at the VULCAN laser and funding via EPSRC. MZ acknowledges the support from the Royal Society.

\section{References}

[1] Pukhov A 2006 Nature Phys. 2439

[2] Gordienko S et al 2004 Phys. Rev. Lett. 93115002 Gordienko S et al 2005 Phys. Rev. Lett. 94103903

[3] Tsakiris G D et al 2006 New J. Phys. 819

[4] Neutze R et al 2000 Nature 406 752-7

[5] Hentschel M et al 2001 Nature 414509

[6] Pellegrini C and Stöhr J 2003 Nucl. Instrum. Methods A 50033

[7] Su Q, Eberly J H and Javanainen J 1990 Phys. Rev. Lett. 64862

[8] Gordienko S et al 2005 Phys. Rev. Lett. 94103903

[9] Schwinger J 1951 Phys. Rev. 82664

[10] Heisenberg W and Euler H 1936 Z. Phys. 98714

[11] Corkum P and Krausz F 2007 Nature Phys. 3381

[12] Carman R L, Forslund D W and Kindel J M 1981 Phys. Rev. Lett. 4629

[13] Bulanov S V, Naumova N M and Pegoraro F 1994 Phys. Plasmas 1745

[14] Lichters R, Meyer-ter-Vehn J and Pukhov A 1996 Phys. Plasmas 33425 Lichters R and Meyer-ter-Vehn J 1997 Multiphoton Processes 1996 (Inst. Phys. Conf. Ser. 154) p 221

[15] Gibbon P 1996 Phys. Rev. Lett. 7650

[16] Einstein A 1905 Ann. Phys. Lpz. 17891

[17] Lopes N C et al 2004 Europhys. Lett. 66 371-7

[18] Bulanov S V et al 2003 Phys. Rev. Lett. 91085001

[19] Zepf M et al 1998 Phys. Rev. E 58 R5253 Tarasevitch et al 2007 Phys. Rev. Lett. 98103902

[20] Baeva T, Gordienko S and Pukhov A 2006 Phys. Rev. E 74046404

[21] Plaja L et al 1998 J. Opt. Soc. Am. B 151904

[22] Watts I et al 2002 Phys. Rev. Lett. 88155001

[23] Norreys P et al 1996 Phys. Rev. Lett. 761832

[24] Mikhailova Y M 2005 JETP Lett. 81571

[25] Gibbon P 2005 Short Pulse Laser Interactions with Matter: An Introduction (London, UK: Imperial College Press) ISBN: 1860941354

[26] Quere F et al 2006 Phys. Rev. Lett. 96125004

[27] Dromey B et al 2006 Nature Phys. 2456

[28] Wilks S C et al 1992 Phys. Rev. Lett. 691383

[29] Geissler M et al 2007 3D simulations of surface harmonic generation with few-cycle laser pulses New J. Phys. 9218

[30] Dromey B et al 2004 Rev. Sci. Instrum. 75 645-8

[31] Doumy G et al 2004 Phys. Rev. E 69026402 Kapteyn H et al 1991 Opt. Lett. 16490

[32] Danson C N et al 2004 Nucl. Fusion 44 S239

[33] Von Hamos L 1939 Z. Kristallogr. 10117

[34] Gales S G and Bentley C D 2004 Rev. Sci. Instrum. 754001 